CWRU-P10-95

astro-ph/9509126

September 1995

\title{
BIG BANG NUCLEOSYNTHESIS CONSTRAINTS ON PRIMORDIAL MAGNETIC FIELDS
}

\author{
Peter J. Kernan, Glenn D. Starkman and Tanmay Vachaspati \\ Department of Physics \\ Case Western Reserve University \\ 10900 Euclid Ave., Cleveland, OH 44106-7079
}

\begin{abstract}
We reanalyze the effect of magnetic fields in BBN, incorporating several features which were omitted in previous analyses. We find that the effects of coherent magnetic fields on the weak interaction rates and the electron thermodynamic functions $\left(\rho_{e}, P_{e}\right.$, and $\left.d \rho_{e} / d T_{\gamma}\right)$ are unimportant in comparison to the contribution of the magnetic field energy density in BBN. In consequence the effect of including magnetic fields in BBN is well approximated numerically by treating the additional energy density as effective neutrino number. A conservative upper bound on the primordial magnetic field, parameterized as $\zeta=2 e B_{r m s} /\left(T_{\nu}^{2}\right)$, is $\zeta \leq 2\left(\rho_{B}<0.27 \rho_{\nu}\right)$. This bound can be stronger than the conventional bound coming from the Faraday rotation measures of distant quasars if the cosmological magnetic field is generated by a causal mechanism.
\end{abstract}




\section{Introduction}

It has frequently been suggested that a significant cosmological magnetic field is present [1, 2, 3] and over the last few years several proposals have been made how to generate such a field [4]. Big bang nucleosynthesis (BBN) provides us with a tool that can be used to probe physics in the early universe and as such is invaluable in the study of primordial magnetic fields. In fact, BBN appears to be the only tool presently available that can explore magnetic fields in the pre-recombination universe and hence the physics that goes into generating the magnetic field.

The effect of magnetic fields on BBN was discussed in the early pioneering works of Greenstein [5] and Matese and O'Connell [6]. The conclusions and understanding in these papers were broadly correct. But now, with advances in our understanding of BBN and faster computers, we can make tighter quantitative estimates of the light element abundances $\left({ }^{4} \mathrm{He}, \mathrm{D}\right.$ and $\left.{ }^{3} \mathrm{He}\right)$. The issue has also been examined in the recent literature, first by Cheng, Schramm and Truran (CST) [7] and subsequently by Grasso and Rubinstein (GR) [8]. Our analysis differs from these calculations in that we have included several factors omitted in these works, corrected some apparent errors and resolved some of the conflicts. We also provide analytic arguments based on the Euler-Maclaurin expansion to deduce the changes in various quantities due to the magnetic field.

There are two primary effects of magnetic fields on BBN : (i) the magnetic field energy density contributes to the cosmological expansion rate, and, (ii) the electron phase space changes due to the magnetic field. A priori, we cannot say if the dominant effect that determines any changes to the light element abundances is (i) or (ii), so it is necessary to go through the full analysis and to evaluate the magnitudes of both effects. The determination of the effects of the electron phase space change are quite involved and it is here that we disagree with CST and GR. However, after the full evaluation is carried out, the dominant effect of the magnetic field turns out to be its direct contribution to the cosmological expansion rate. The computationally

expensive contributions to the weak rates and the electron thermodynamic 
functions can be neglected, and in practice the numerical BBN computation can be avoided altogether by expressing the bound on the magnetic field energy density as an equivalent bound on the effective number of neutrinos, a number commonly derived in the BBN literature. This should be contrasted with the claims of GR who find that the change in $\rho_{e}$ dominates, and CST who find that the changes in the weak interaction rates dominate. We agree with the original conclusions of Matese and O'Connell.

Although a rigorous comparison of the effects (i) and (ii) can only be made after a systematic evaluation, one way to understand why the effect on the expansion rate is more important than the change in the interaction rates is to note that the expansion rate changes in proportion to $B^{2}$ while the interaction rates change in proportion to $\alpha B^{2}$, with $\alpha \simeq 1 / 137$ being the fine structure constant. Any other numerical factors occuring in the interaction rates turn out not to be large enough to overcome the suppression by $\alpha$.

Our analysis assumes a magnetic field that scales as:

$$
B \propto R^{-2} \equiv b T_{\nu}^{2}
$$

where $R$ is the scale factor of the universe and $T_{\nu}$ the neutrino temperature. Our analysis also assumes that the cosmological expansion is well described by the Friedmann-Robertson-Walker (FRW) model. The latter assumption is only justified if the magnetic field is sufficiently tangled on scales smaller than the horizon - otherwise we would need to consider the anisotropic expansion of the universe. At the same time, the assumption in (1) is valid only on scales large enough for the plasma conductivity to keep the magnetic field frozen-in. (Today this frozen-in scale is of order $10^{13} \mathrm{cms}$.) Recent work by Jedamzik et. al. [15] considers the magnetohydrodynamical evolution of the system and concludes that the magnetic field can dissipate on yet larger scales which would correspond to scales of several megaparsecs today. The $\mathrm{BBN}$ constraints that we derive on primordial magnetic fields are therefore only valid for magnetic fields that are coherent on scales larger than the scale on which magnetic fields can dissipate and smaller than the horizon scale. In particular, magnetic fields generated during inflation are likely to be coherent on super-horizon scales and have not been fully dealt with by our analysis. 
As the dominant effect of magnetic fields on BBN is due to the contribution of the field to the energy density, it will be convenient to express the energy density in magnetic fields in terms of the number of equivalent additional neutrino species. The energy density contributed by the magnetic field is

$$
\rho_{B}=\frac{B^{2}}{8 \pi}
$$

where, $B^{2}$ is the volume average of $|\vec{B}(\vec{x})|^{2}$. The contribution of $N_{\nu}$ light $(m \ll 1 \mathrm{MeV})$ neutrino species to the energy density of the universe is

$$
\rho_{\nu}=\frac{7 \pi^{2}}{120} N_{\nu} T_{\nu}^{4}
$$

and so $\rho_{B}$ may be written as a number of equivalent additional neutrino species

$$
\Delta N_{\nu}^{B}=\frac{15}{7 \pi^{3}} b^{2},
$$

where $b=B / T_{\nu}^{2}$ is the constant which we seek to constrain. This gives

$$
\rho_{\nu}+\rho_{B}=\left(N_{\nu}+\Delta N_{\nu}^{B}\right) \rho_{\nu}\left(N_{\nu}=1\right) .
$$

For standard BBN $N_{\nu}=3$. Krauss and Kernan (KK) argue that a very conservative bound on $\Delta N_{\nu}$ is $\Delta N_{\nu}<.8$ [9], leading to the bound $b<3.4$.

The bound in $\mathrm{KK}$ on $N_{\nu}$ is derived by requiring that at least $5 \%$ of 1000 Monte Carlo runs simultaneously satisfy the abundance constraints $\mathrm{D}+{ }^{3} \mathrm{He} / \mathrm{H}<10^{-4}$ and $Y_{p}<.25$, for any value of $\eta$, the baryon to photon ratio, for a given number of neutrinos. The random variables in the the BBN Monte Carlo uses 11 nuclear reaction rates and the neutron lifetime as random variables. The result, $N_{\nu}<3.8$, is conservative in that the abundance limits assumed allow for generous systematic errors in the observed ${ }^{4} \mathrm{He} / \mathrm{H}$ abundance in HII regions, and the likelihood of anomalous presolar abundances of $\mathrm{D}$ and ${ }^{3} \mathrm{He}$.

In the following sections we lay the physics groundwork for our bound on primordial magnetic fields. First we consider the effects of the $B$ field on the electron phase space, then the resultant changes in the weak interaction rates and the time-temperature relation. Finally we tie these all together and numerically solve for the neutron fraction as a function of temperature. 


\section{Electron Phase Space}

The dispersion relation for the electron in the presence of a uniform magnetic field is

$$
E=\left(p_{e}^{2}+m_{e}^{2}+e B(2 n+s+1)\right)^{1 / 2}
$$

with $e$ the electron charge, $B$ the magnetic field strength, and $n$ the principal, and $s$ the magnetic quantum number of the Landau level. This relation is valid for a magnetic field that is small compared to the critical field $B_{c}=m_{e}^{2} / e$ and has only been derived at zero temperature. For stronger fields and at high temperatures we expect further corrections to this formula. For example, the next order correction would be to include the anomalous magnetic moment of the electron. However, the analyses available in the literature [10] indicate that these higher order corrections generally have a very small effect at the magnetic field strengths and temperatures of interest and so we will only consider the dispersion relation in (6) throughout this paper. The full treatment of the problem is computationally impractical until an analytic approximation is developed for $E(n, s, p, B)$.

After summing over the electron spin in (6) the zero-chemical potential phase space of electrons appearing in weak interaction rates and electron thermodynamic functions changes according to

$$
2 \frac{d^{3} p}{(2 \pi)^{3}} f_{F D}\left(E_{0}\right) \longrightarrow \sum_{n=0}^{\infty}\left(2-\delta_{n 0}\right) \frac{d p_{e}}{2 \pi} \frac{e B}{2 \pi} f_{F D}\left(E_{B}, T\right)
$$

with $E_{B}^{2}=p_{e}^{2}+m_{e}^{2}+2 e B n$ and $f_{F D}(E, T)$ the Fermi-Dirac distribution at temperature $\mathrm{T}$ :

$$
f_{F D}(E, T)=\frac{1}{1+\exp (E / T)} .
$$

We have neglected the chemical potential, $\phi$ of the electrons since $\phi / T_{\gamma}$ is of order the baryon to photon ratio, $\eta$, which is small $\left(\mathrm{O}\left(10^{-10}\right)\right)$; hence any $B$ and $\phi$-dependent correction to the standard result would be second order in small quantities. The electron thermodynamic functions are given by the usual prescription of integrating the relevant quantity over phase space. Thus 


$$
\begin{aligned}
n_{e} & =\frac{e B}{(2 \pi)^{2}} \sum_{-\infty}^{+\infty}\left(2-\delta_{n 0}\right) \int_{-\infty}^{+\infty} f_{F D}\left(E_{B}, T_{\gamma}\right) d p_{z} \\
\rho_{e} & =\frac{e B}{(2 \pi)^{2}} \sum_{-\infty}^{+\infty}\left(2-\delta_{n 0}\right) \int_{-\infty}^{+\infty} E_{B} f_{F D}\left(E_{B}, T_{\gamma}\right) d p_{z} \\
P_{e} & =\frac{e B}{(2 \pi)^{2}} \sum_{-\infty}^{+\infty}\left(2-\delta_{n 0}\right) \int_{-\infty}^{+\infty} \frac{E_{B}^{2}-m_{e}^{2}}{3 E_{B}} f_{F D}\left(E_{B}, T_{\gamma}\right) d p_{z}
\end{aligned}
$$

$n_{e}$ does not appear in the time-temperature relation, but is useful for illustrative purposes, e.g. the perhaps counter-intuitive result that the electron number density increases as the magnetic field increases. This is easy to show by expanding (9) in an Euler-MacLaurin expansion.

$$
\begin{aligned}
n_{e} & \simeq \frac{T_{\gamma}^{3}}{4 \pi^{2}}\left[4 \int_{0}^{\infty} \frac{\rho^{2} d \rho}{1+e^{\sqrt{\rho^{2}+m_{e}^{2} / T_{\gamma}^{2}}}}\right. \\
& +\frac{\left(\zeta r_{\nu \gamma}\right)^{2}}{24} \int_{0}^{\infty} \frac{d \eta}{\sqrt{\eta^{2}+m_{e}^{2} / T_{\gamma}^{2}}} \frac{1}{1+\cosh \sqrt{\eta^{2}+m_{e}^{2} / T_{\gamma}^{2}}} \\
& \left.+\mathcal{O}\left(\left(\zeta r_{\nu \gamma}\right)^{4}\right)\right]
\end{aligned}
$$

where $\zeta=2 e B / T_{\nu}^{2}=2 e b$, and $r_{\nu \gamma}=\left(T_{\nu} / T_{\gamma}\right)^{2}$. Although we will quote limits on $b$ or $\zeta$, which are constants, we will also use $z=\zeta r_{\nu \gamma}$, which is what usually appears in the equations. Clearly from (13),

$$
\lim _{B \rightarrow 0} \frac{d n_{e}}{d B}>0
$$

The interpretation is that there are two effects of increasing the magnetic field strength on the electron phase space -1 ) the energy cost of populating $n>0$ levels becomes greater due to the larger spacing of the Landau levels, and, 2) the areal element in the phase space decreases due to the decreased crosssectional area of each Landau level. The second effect is stronger than the first and causes the number density of electrons to increase with $B$. For $\rho_{e}$, 
the sign of the effect is less clearcut (indeed it depends on the temperature):

$$
\begin{aligned}
\rho_{e} & \simeq \frac{T_{\gamma}^{3}}{4 \pi^{2}}\left[4 \int_{0}^{\infty} \frac{\rho^{2} \sqrt{\rho^{2}+m_{e}^{2} / T_{\gamma}^{2}} d \rho}{1+e^{\sqrt{\rho^{2}+m_{e}^{2} / T_{\gamma}^{2}}}}\right. \\
& +\frac{z^{2}}{12} \int_{0}^{\infty} d \eta\left(\frac{1}{2\left(1+\cosh \sqrt{\eta^{2}+m_{e}^{2} / T_{\gamma}^{2}}\right)}-\frac{1 / \sqrt{\eta^{2}+m_{e}^{2} / T_{\gamma}^{2}}}{1+e^{\sqrt{\rho^{2}+m_{e}^{2} / T_{\gamma}^{2}}}}\right) \\
& \left.+\mathcal{O}\left(z^{4}\right)\right]
\end{aligned}
$$

The first term inside the square brackets gives the electron energy density when $B=0$. The second term is the correction to the energy density due to the presence of a magnetic field and can be negative or positive, depending on the temperature $T_{\gamma}$.

Assuming that the integral in the $z^{2}$ term of (15) is $\mathcal{O}(1)$, it is clear that the change in the energy density of electrons in the field is smaller than the pure magnetic field contribution to the energy density. Since the former is due to coupling of the magnetic field to the electrons, it is proportional to $z^{2} \propto \alpha B^{2}$ times small numerical factors of order $0.01-0.1$. This is smaller by $\mathcal{O}(\alpha)$ than the $B^{2} / 8 \pi$ term. We expect the phase space corrections to the weak interaction rates to also be of order $z^{2}$ times small numerical factors.

In [8], only the changes in $\rho_{e}$ were taken into account; but the changes in $P_{e}$ and $d \rho_{e} / d T_{\gamma}$ must also be taken into account since they appear in the BBN time-temperature relation. We will spare the reader the explicit formula for $d \rho_{e} / d T_{\gamma}$ which is quite a lengthy expression with many extra terms involving $d B / d T_{\gamma}$.

\section{Neutron Fraction}

The binding energy per nucleon of helium-4, the most strongly bound light nucleus, is $7.1 \mathrm{MeV}$. Yet, well below this temperature the abundance of ${ }^{4} \mathrm{He}$ compared to hydrogen is quite small, because the high energy tail of the photon distribution breaks up any deuteron $(Q=2.2 \mathrm{MeV})$ which forms. At

a (slightly $\Omega_{B} h_{0}^{2}$ dependent ) temperature of $T_{\gamma} \simeq .07 \mathrm{MeV}$ the high energy tail of the photon distribution is no longer energetic enough to break up all 
the deuterons. When this "deuterium bottleneck" is passed, all the available neutrons are swiftly incorporated into ${ }^{4} \mathrm{He}$, with trace amounts of other light nuclei, D, ${ }^{3} \mathrm{He}$ and ${ }^{7} \mathrm{Li}$ produced. To a very good approximation, $Y_{p}$, the primordial ${ }^{4} \mathrm{He}$ mass fraction is given by twice the neutron fraction at the breaking of the deuterium bottleneck. Thus to discover the importance of the effects of the corrections to the BBN dynamics, for a first pass it is sufficient to examine the evolution of the neutron fraction with all strong interations turned off.

With respect to comparison with observed abundances the ${ }^{4} \mathrm{He}$ production is the most likely to be noticeably affected by the changes in BBN dynamics in the presence of the magnetic field. This is because the ${ }^{4} \mathrm{He}$ observations are sensitive at the $5 \%$ level or better, whereas all the other observations are sensitive only at the $50-100 \%$ level. Also, changes induced in the nuclear reaction rates when they are important $\left(T_{\gamma} \leq 0.07 \mathrm{MeV}\right)$ are likely to be much smaller than those induced in the weak interaction rates when they are important $\left(T_{\gamma} \gg 0.07 \mathrm{MeV}\right)$, both because the electron magnetic moment is much larger than the nuclear magnetic moment, and because the magnetic field decreases as $T_{\nu}^{2} \simeq T_{\gamma}^{2}$, and is therefore small by the time the nuclear reactions take place. Finally, the other elements are sensitive to changes in the time-temperature relation due to the magnetic field only because of the very small change in the temperature at which their number density falls out of quasi-equilibrium, whereas the neutron fraction at the breaking of the deuterium bottleneck (and hence the ${ }^{4} \mathrm{He}$ abundance) is sensitive to the change in the equilibrium neutron fraction during weak equilibrium, the change in the departure of the actual neutron fraction from it equilibrium value, the change in the freezeout temperature of the weak interactions, the change in the fraction of neutrons which decay between weak freezeout and the breaking of the bottleneck, and the change in the temperature at which the deuterium bottleneck breaks.

The neutron fraction evolves according to

$$
\frac{d X_{n}}{d t}=-\lambda_{n p} X_{n}+\lambda_{p n}\left(1-X_{n}\right)
$$

where $\lambda_{n p}$ is the rate per nucleon of neutron conversions to protons and $\lambda_{p n}$ 
is the rate of proton conversions to neutrons.

$$
\lambda_{n p}=\lambda_{n \rightarrow p e^{-} \nu}+\lambda_{n e^{+} \rightarrow p \nu}+\lambda_{n \bar{\nu} \rightarrow p e^{-}}
$$

with a corresponding formula for $\lambda_{p n}$. For the numerical calculation we left out the 3-body reaction, $p e^{-} \nu \rightarrow n$ which never becomes important. If the weak interactions were in equilibrium, which would be the case if the interaction rates were much faster than the Hubble expansion rate, then the neutron fraction would assume its equilibrium value

$$
X_{n}^{e q}=\frac{\lambda_{p n}}{\lambda_{n p}+\lambda_{p n}}
$$

The weak interaction rates at these low energies are given simply by integrals of $p_{e} E_{e} E_{\nu}^{2}$ over the available phase space. For example,

$$
\lambda_{n \rightarrow p e^{-} \nu}=\frac{G_{F}^{2} T_{\gamma}^{2}\left(g_{V}^{2}+3 g_{A}^{2}\right)}{(2 \pi)^{3}} z \sum_{n=0}^{n_{c}}\left(2-\delta_{n 0}\right) \int_{0}^{p_{m}} d p_{z} E_{\nu}^{2} g\left(E_{e} / T_{\gamma}\right) g\left(E_{\nu} / T_{\nu}\right)
$$

Here $G_{F}$ is the Fermi decay constant, $g_{V}$ and $g_{A}$ are the vector and axialvector coupling constants. The electron energy is given by

$$
E_{e}=\left(p_{e}^{2}+m_{e}^{2}+2 e B n\right)^{1 / 2}
$$

the neutrino energy by

$$
E_{\nu}=\Delta-E_{e}
$$

with $\Delta=1.293 \mathrm{MeV}$ being the neutron-proton mass difference. The sum in (19) is taken to a maximum of $n_{c}=\left[\left(\Delta^{2}-m_{e}^{2}\right) / 2 e B\right]$ (where $[x]$ is the largest integer $\leq x)$, and the momentum integral is up to $p_{m}=\sqrt{\Delta^{2}-m_{e}^{2}-2 n e B}$. $g$ is the Fermi blocking factor and is given by:

$$
g(E / T) \equiv 1-f_{F D}(E / T)=(\exp (-E / T)+1)^{-1} .
$$

The other weak interaction rates have a similar form, with appropriate factors of $f_{F D}$ and $g$ in the integrands, and with the limits on the sum and integral 
given by the requirement of positive energy for the electron and neutrino. For example,

$$
\lambda_{n \nu \rightarrow p e^{-}}=\frac{G_{F}^{2} T_{\gamma}^{2}\left(g_{V}^{2}+3 g_{A}^{2}\right)}{(2 \pi)^{3}} z \sum_{n=0}^{\infty}\left(2-\delta_{n 0}\right) \int_{0}^{\infty} d p_{z} E_{\nu}^{2} g\left(E_{e} / T_{\gamma}\right) f\left(E_{\nu} / T_{\nu}\right)
$$

With the exception of beta decay we find that the magnetic field effects on the electron phase space decreases all the weak rates. For small $z$ we can see this once again using the Euler-McLaurin expansion:

$$
\begin{aligned}
\lambda_{n \nu \rightarrow p e^{-}} & =\frac{G_{F}^{2} T_{\gamma}^{2}\left(g_{V}^{2}+3 g_{A}^{2}\right)}{(2 \pi)^{3}}\left(2 \int_{0}^{\infty} d x \int_{p_{\min }(x)}^{\infty} G_{-}\left(E_{e}\left(p_{z}, x\right)\right) d p_{z}\right. \\
& \left.-\frac{z^{2} T_{\gamma}^{2}}{12} \int_{p_{\min }(0)}^{\infty}\left[\frac{1}{E_{e}} \frac{d G_{-}\left(E_{e}\right)}{d E_{e}}\right]_{x=0} d p_{z}\right)
\end{aligned}
$$

where

$$
\begin{gathered}
E_{e}\left(p_{z}, x\right)=\left(p_{z}^{2}+m_{e}^{2}+x\right)^{1 / 2}, \\
p_{\text {min }}(x)=\left(\Delta^{2}-m_{e}^{2}-x T_{\gamma}^{2}\right)^{1 / 2}
\end{gathered}
$$

and

$$
G_{-}\left(E_{e}\right)=\left(E_{e}-\Delta\right)^{2} \frac{1}{1+e^{\left(E_{e}-\Delta\right) / T_{\nu}}} \frac{1}{1+e^{-E_{e} / T_{\gamma}}} .
$$

Thus

$$
\begin{aligned}
{\left[\frac{1}{E_{e}} \frac{d G_{-}\left(E_{e}\right)}{d E_{e}}\right] } & =\frac{E_{e}-\Delta}{E_{e}} \frac{1}{1+e^{\left(E_{e}-\Delta\right) / T_{\nu}}} \frac{1}{1+e^{-E_{e} / T_{\gamma}}} \\
& \times\left[2-\frac{E_{e}-\Delta}{T_{\nu}} \frac{1}{1+e^{-\left(E_{e}-\Delta\right) / T_{\nu}}}+\frac{E_{e}}{T_{\gamma}} \frac{1}{1+e^{E_{e} / T_{\gamma}}}\right] .
\end{aligned}
$$

We see that only the second term in the expression in square brackets is negative and that (for $x=0$ ), by the time $E_{e}$ is large enough for the term to dominate over the other two terms in the square brackets, the prefactor is exponentially suppressed. The coefficient of the $z^{2}$ term of (24) is therefore negative, and the B-field decreases this rate. Similar arguments can be applied to the other $2 \rightarrow 2$ rates. Though the conclusions are not always so clear analytically, our numerical studies show a decrease in all the 2-body rates for $B>0$. 
For beta decay the momentum cutoff introduces an $n$ cutoff, and with $B>B_{c}=\left(\Delta^{2}-m_{e}^{2}\right) / 2 e$ only the $n=0$ term survives in the sum. Thus it is easily seen that $\lambda_{n \rightarrow p e^{-} \nu}$ increases linearly with $B$ for $B \geq B_{c}$. We find numerically that it also increases (though not linearly) for $B<B_{c}$.

There are three competing effects for the weak interactions. At high temperatures the weak rates are slower. This by itself does not imply anything about the equilibrium neutron fraction, since the neutron fraction (see (18)) depends on a ratio of rates; but, because $\lambda_{p n}$ decreases more than $\lambda_{n p}$, there is a small decrease in the equilibrium neutron fraction (see Figure 1). This is the first effect. However, below about $1 \mathrm{MeV}$, the weak interaction rates are too slow to maintain this equilibrium. Since $X_{n}^{e q}$ is decreasing with temperature, the actual neutron fraction lags behind, and is therefore always greater than, the equilibrium value. Because the $\mathrm{B}$ field lowers the interactions rates, $X_{n}$ is less able to track $X_{n}^{e q}$, and so lags behind the dropping equilibrium neutron fraction more than usual. This second effect tends to increase the neutron fraction at a given temperature. Finally at later times, $T_{\gamma} \lesssim .2 \mathrm{MeV}$, neutron decay dominates the weak interaction rates. The $B$ field causes the neutron decay rate to increase, lowering the neutron fraction. However, since neutron decay is the dominant reaction only at lower temperatures, the $B$ field has already decreased significantly, and the change in the decay rate is negligible.

If these changes in the reaction rates were the only effects, then the decline in the equilibrium fraction and the increase in the neutron decay rate at low temperatures would outweigh the increased departure from equilibrium (due to the lower reaction rates) at high temperatures. The net result would be a lower neutron fraction and hence a lower ${ }^{4} \mathrm{He}$ abundance as can be seen from the dotted line in Figure 2.

However, as we shall discuss in detail below, these are not the dominant effects. 


\section{Time-Temperature Relation}

To solve for BBN abundances we must follow not just the evolution of the neutron fraction, but also the evolution of the photon and neutrino temperatures with time. Initially, $T_{\gamma} \geq 5 \mathrm{MeV}$, the neutrinos and electromagnetic fluids are assumed to be in perfect thermal contact and $T_{\gamma}^{i}=T_{\nu}^{i}$. Later, the photon and neutrino temperatures evolve differently due to electron-positron annihilation [11, 12], which heats the photons, but not the neutrinos. This assumes zero net energy exchange between the neutrino and electromagnetic fluids, a very good approximation for BBN [13]. Assuming that the neutrinos are decoupled well before this annihilation takes place, $T_{\nu}$ evolves as the inverse scale factor

$$
R T_{\nu}=\text { constant }
$$

For the photons we use energy conservation in the electromagnetic plasma.

$$
d\left[\left(\rho_{e m}+P_{e m}\right) R^{3}\right]=R^{3} d P_{e m},
$$

with $\rho_{e m}=\rho_{e}+\rho_{\gamma}$ and $P_{e m}=P_{\gamma}+P_{e}$. It follows that

$$
d \rho_{e m} / d T_{\gamma}=-3\left(\rho_{e m}+P_{e m}\right) R^{-1} d R / d T_{\gamma}
$$

The magnetic field energy density is not to be included in (31). This would be appropriate only if the large scale $B$ field was in thermal equilibrium with the electromagnetic fluid, and hence evolved according to $T_{\gamma}$ rather than the inverse scale factor.

The Hubble expansion law for the radiation dominated early universe is

$$
H \equiv \frac{1}{R} \frac{d R}{d t}=\sqrt{\frac{8 \pi G \rho_{T}}{3}}
$$

with $\rho_{T}$ representing all forms of energy density in the universe. (Note that the magnetic field energy is included here but not in (31)). From (31) and (32) we obtain the time-temperature relation which we are seeking,

$$
\frac{d T_{\gamma}}{d t}=-3 H \frac{\rho_{e m}+P_{e m}}{d \rho_{e m} / d T_{\gamma}} .
$$


The analogous equation for the neutrino temperature, from (29) and (32) is

$$
\frac{d T_{\nu}}{d t}=-H T_{\nu}
$$

The complication in solving (33) in the case of a magnetic field is that $\rho_{e}$ depends both on $T_{\gamma}$ and on $B=b T_{\nu}^{2}, \rho_{e}=\rho_{e}\left(T_{\gamma}, T_{\nu} ; \zeta\right)$. We therefore write

$$
\frac{d \rho_{e}}{d T_{\gamma}}=\frac{\partial \rho_{e}}{\partial T_{\gamma}}+\frac{\partial \rho_{e}}{\partial T_{\nu}} \frac{d T_{\nu}}{d T_{\gamma}}
$$

If we now define

$$
\frac{\partial \rho_{e}}{\partial T_{\gamma}} \equiv h\left(T_{\gamma}, T_{\nu}\right), \quad \frac{\partial \rho_{e}}{\partial T_{\nu}} \equiv \frac{1}{T_{\nu}} j\left(T_{\gamma}, T_{\nu}\right)
$$

we can rewrite (33) Using (34) and (35) as,

$$
\frac{d T_{\gamma}}{d t}=-3 H \frac{\rho_{e m}+P_{e m}}{d \rho_{\gamma} / d T_{\gamma}+h}\left(1-\frac{j}{3\left(\rho_{e m}+P_{e m}\right)}\right) .
$$

The right-hand sides of equations (34) and (37) depend only on $T_{\nu}, T_{\gamma}$, and the constant $\zeta$ (or equivalently $b$ ) and can be integrated together to obtain $T_{\nu}(t)$ and $T_{\gamma}(t)$.

Although the details must be obtained numerically, the basic picture is clear. The increased energy density due to the $B^{2} / 8 \pi$ term means that at any given temperature $\left(T_{\gamma}\right.$ or $\left.T_{\nu}\right)$ the universe is younger and expanding faster than in the standard scenario $(B=0)$. This has two important consequences. First, the weak interaction rates, which are already slower than in the $B=0$ case have an even harder time maintaining the equilibrium neutron fraction. Since the equilibrium neutron fraction is dropping, this increased lag will cause the actual neutron fraction to be higher than for $B=0$. Second, the time between weak interaction freezeout and the breaking of the deuterium bottleneck is shortened. Thus there is less time for the extra neutrons to decay than if $B$ were zero. This also acts to increase the final neutron fraction. Although there is a lowering of the temperature at which the bottleneck is 
broken, this is a second order effect [14] and the change in this temperature turns out to be only about $0.1 \%$ and is therefore ignored.

The net result is that if one takes into account only the change in the time-temperature relation, the final neutron fraction rises and so does the ${ }^{4} \mathrm{He}$ abundance.

\section{Results}

We are left to determine whether the neutron fraction rises because of the change in the time-temperature relation, or falls because of the changes in the weak interaction rates. We find that the effects of the change in the time-temperature relation from the magnetic field energy density are much larger than those in the time-temperature relation or the weak interaction rates due to the changes in the electron phase space. We have not been able to demonstrate this convincingly using only analytic methods; however, basically it is because the changes in the phase space are proportional to $\alpha B^{2}$, whereas the change in the energy density is proportional to simply to $B^{2}$ (with no $\alpha$ ).

We integrated the coupled differential equations (34), (37) and (16) to obtain the photon and neutrino temperatures and the neutron fraction as a function of time. We ran the computer code from $T_{\gamma}=5 \mathrm{MeV}$ to $.07 \mathrm{MeV}$, when the deuterium bottleneck is broken and almost all the remaining neutrons are converted to ${ }^{4} \mathrm{He}$. We did this for several values of $\zeta=2 e B / T_{\nu}^{2}$.

In Figure 2 we display the evolution of the neutron fractions for $\zeta=0$, and for $\zeta=2$ in each of five different limits. The long dashed curve shows the $\zeta=0$ case; the solid curve shows $X_{n}$ with the full calculation; in the dotted curve we have turned on only the dependence of the weak interaction rates on the magnetic fields; in the short-dash curve we have only turned on the dependence on the magnetic fields only of the electron thermodynamic functions; and with the dash-dotted curve we have only turned on the dependence of the expansion rate on the energy density of the magnetic field. It is clear that the latter is by far the dominant effect. The change in $X_{n}\left(T_{\gamma}=.07 \mathrm{MeV}\right)$ from the canonical case, $B=0$, is $-2.0 \times 10^{-5}$ for 
weak interactions only, $<5.0 \times 10^{-6}$ for electron thermodynamics only and $+4.8 \times 10^{-3}$ for magnetic field energy density only. Thus the limit on B is equivalent to one on the number of neutrinos, and for $N_{\nu} \leq 3.8$, our limit on $B$ is given by,

$$
\zeta \leq 2
$$

In units of the neutrino energy density this can also be expressed as

$$
\rho_{B} \leq .27 \rho_{\nu},
$$

where $\rho_{\nu}$ is the energy density contributed by the standard 3 light $(<<1$ $\mathrm{MeV}$ ) neutrinos.

An additional point of interest is that the change in the electron thermodynamics during the era of $e^{+} e^{-}$annihilation causes a slight decrease in $T_{\nu} / T_{\gamma}$ with respect to the canonical value, $(4 / 11)^{1 / 3}$. This can be understood heuristically as follows. The magnetic field increases the electron number density. This is equivalent to some fraction of an additional degree of freedom residing in the electromagnetic fluid in the early universe. When the $e^{+} e^{-}$pairs annihilate the additional entropy is transferred to the photons, thus "heating" them slightly more with respect to the neutrinos. For the values of the magnetic field allowed by BBN the change is small enough that it has little effect on other cosmological parameters which depend on the neutrino temperature, such as the mass of a light neutrino required for $\Omega_{\nu}=1$. If very large magnetic fields were allowed, for example, with $\zeta=8.0$ (16.0), we find

$$
\frac{\Delta\left(T_{\nu} / T_{\gamma}\right)}{T_{\nu} / T_{\gamma}}=-1.7 \%(-5.6 \%),
$$

and this effect becomes significant.

In Figure 3 we display the evolution of the neutron fraction as a function of $T_{\gamma}$ from $T_{\gamma}=3$ to $T_{\gamma}=0.07$ for $\zeta=.1,2,4$. The points on the curve where $t=.1,1,10$ and 100 seconds are marked. The two dominant effects which we described are manifest. At high temperature, the $2 \rightarrow 2$ weak interactions are dominant. The higher the $\mathrm{B}$ field the faster the universe expands at a given $T_{\gamma}$, and the more the actual neutron fraction lags behind 
the decreasing equilibrium abundance. At low temperatures, neutron decay is dominant. The higher the B field, the sooner the universe cools sufficiently to break the deuterium bottleneck, so the sooner neutron decay terminates and the remaining neutrons are converted almost instantaneously to ${ }^{4} \mathrm{He}$. This is seen by the earlier termination of the $X_{n}$ line. Both these effects lead to a higher neutron fraction.

\section{Discussion}

Our analysis of the effects of magnetic fields on BBN have allowed us to place constraints on the field strength present at the BBN epoch. The analysis is based on the following assumptions:

a) The cosmological model is an FRW model. This means that the magnetic field does not lead to anisotropic expansion of the universe. For this the magnetic field should not be coherent on the scale of the horizon such as happens in proposals that generate magnetic fields from inflation. However, it seems likely that if the magnetic fields are not too strong, the constraint we have derived using an isotropic FRW universe should still apply. If there are significant departures from FRW universes, the magnetic field would be constrained by measurements of the isotropy of the universe stemming from measurements of the microwave background [17].

b) The magnetic field is assumed to have a scaling given by (1) and hence we have assumed that it does not dissipate. The shortest scale that does not dissipate has been argued to be given by the magnetic Jeans length [15]. Another scale that is probably relevant is the scale on which the magnetic fields are frozen in due to the high electrical conductivity of the cosmological plasma [1, 2].

c) The dispersion relation is assumed to be given by (6) which is known to be incorrect when the magnetic field is strong (compared to $m_{e}^{2} / e$ ) or at high temperatures. But current analyses [10] indicate that the corrections are small in the range of temperatures and magnetic field strengths that are relevant for us. We assume that these corrections will not change our result significantly. This assumption can be removed once we have a better analytic 
grasp of the corrections to the dispersion relation as it would be computationally infeasible to use the high magnetic field strength and temperature dispersion relation that is known in its present form.

Within the framework of these assumptions, we have derived a BBN constraint on the present cosmological volume averaged magnetic field strength $B_{r m s}$ (equivalently, on the magnetic field energy density) defined by

$$
B_{r m s}^{2}=\frac{1}{V} \int_{V} d^{3} x|\vec{B}(\vec{x})|^{2}
$$

Using (38) and the evolution equation (11), the constraint is:

$$
B_{r m s}<10^{-6} G \text {. }
$$

We would like to emphasize that this constraint is a local constraint and not a constraint on the magnetic field strength at any particular coherence scale.

An oft quoted constraint on primordial magnetic fields comes from the Faraday rotation measures of distant quasars. This constraint is

$$
\bar{B}<10^{-9} G
$$

where $\bar{B}$ is the line-averaged magnetic field:

$$
\bar{B}=\frac{1}{L} \int_{\Gamma} d \vec{s} \cdot \vec{B}(\vec{x})
$$

and the curve $\Gamma$ is the line of sight (null geodesic) of length $L$ from the quasar to the earth.

To compare (41) with (42), we need to know something about the spectrum of the magnetic field [16]. If the field is homogenous on the quasar distance scale, then a direct comparison can be made and we see that the $\mathrm{BBN}$ constraint is much weaker. This comparison is relevant if the magnetic fields arise from inflation where it is assumed to be coherent on horizon scales (subject to the discussion in point ( $a$ ) above). If, on the other hand, a causal mechanism is found to generate a magnetic field, the field is likely to be coherent on scales that are much smaller than the horizon, say on a scale $\xi$. 
For example, in the case of magnetic fields generated in cosmological phase transitions, we expect 16.

$$
B_{r m s} \simeq \sqrt{\frac{L}{\xi}} \bar{B}
$$

and the Faraday rotation constraint in (42) translates into

$$
B_{r m s}<\sqrt{\frac{L}{\xi}} 10^{-9} G
$$

The BBN constraint (41) can therefore be much stronger than the Faraday rotation constraint if $L / \xi$ is large.

Finally we would like to project the results of this paper from a different viewpoint. This is that the current astrophysical constraints on magnetic fields constrain the large scale magnetic field strength. That is, they say something about the Fourier modes with wavelengths comparable to quasar distances. BBN, on the other hand, provides a complementary tool for constraining the magnetic field energy density which includes contributions from all wavelengths. And for causal mechanisms that generate magnetic fields, BBN is likely to probe the magnetic field spectrum at the smallest wavelength while the Faraday rotation measure is mostly sensitive to wavelengths of order the quasar distance scale.

\section{Acknowledgements}

The authors wish to thank Lawrence Krauss for discussions during the early stages of this work. P.K. and T.V. thank the Aspen Center for Physics for hospitality while this work was in progress.

\section{References}

[1] E.N. Parker, Cosmical Magnetic Fields: Their Origin and Their Activity, Oxford University Press (1979)

[2] Ya.B. Zeldovich, A.A. Ruzmaikin and D.D. Sokoloff, Magnetic Fields in Astrophysics, Gordon and Breach Publishers (1983) 
[3] P. Kroneborg, Rep.Prog.Phys. 57,325 (1994)

[4] M.S. Turner and L.M. Widrow, Phys.Rev.D. 37, 2743 (1988); T. Vachaspati, Phys.Lett.B. 265, 258 (1991); B. Ratra, Ap.J. 391, L1 (1992); Phys.Rev.D. 45, 1913 (1992); A.D. Dolgov, Phys.Rev.D. 48, 2499 (1993); A.D. Dolgov and J. Silk, Phys.Rev.D. 45, 1913 (1992); M. Gasperini, M. Giovannini and G. Veneziano, hep-th/9504083 (1995); A.C. Davis and K. Dimopoulos, CERN-TH/95-175 (1995); G. Baym, D. Bödecker and L. MacLerran, HEP-MINN-95-1344 (1995)

[5] G. Greenstein, Nature 223, 938 (1969).

[6] J.J. Matese and R.F. O'Connell, Ap.J. 160, 451 (1970)

[7] B. Cheng, D.N. Schramm and J.W. Truran, Phys.Rev.D. 49, 5006 (1994)

[8] D. Grasso and H.R. Rubinstein, Astropart.Phys.3,95 (1995)

[9] L.M. Krauss and P.J. Kernan, Phys.Lett.B 347,347 (1995)

[10] R. Geprägs, H. Riffert, H. Herold, H. Ruder and G. Wunner, Phys.Rev.D 49,5582 (1994)

[11] S. Weinberg, Gravitation and Cosmology, John Wiley \& Sons, Inc. (1972)

[12] E.W. Kolb and M.S. Turner, The Early Universe, Addison-Wesley Publishing Company (1990)

[13] S. Dodelson and M.S. Turner, Phys.Rev.D46,3372 (1992)

[14] R. Esmailzadeh, G. Starkman, S. Dimopoulos, ApJ 378,504 (1991)

[15] K. Jedamzik, V. Katalinic and A.V. Olinto (private communication).

[16] K. Enqvist and P. Olesen, Phys. Lett. B319, 178-185,1993.

[17] M.S. Madsen, M.N.R.A.S. 237, 109 (1989). 


\section{Figure Captions}

Figure 1: The equilibrium neutron fraction $X_{n}^{e q}$ as a function of the photon temperature $T_{\gamma}$ for several choices of the magnetic field evolution parameter $\zeta$.

Figure 2: For $\zeta=2$, the neutron fraction $X_{n}$ near the nucleosynthesis temperature region, $\mathcal{O}(70) \mathrm{keV}$, is shown for separate pieces of the full calculation. The changes from the electron thermodynamic functions, $\left(\rho_{e}, P_{e}, d \rho_{e} / d T_{\gamma}\right)$, the weak interaction rates $\lambda_{n \leftrightarrow p}$, and the magnetic field energy density $\rho_{B}$, on the neutron fraction are shown individually. Also plotted is the neutron fraction with all/none of the above corrections.

Figure 3: The neutron fraction $X_{n}$ as a function of the photon temperature $T_{\gamma}$ for several choices of the magnetic field evolution parameter $\zeta$. 


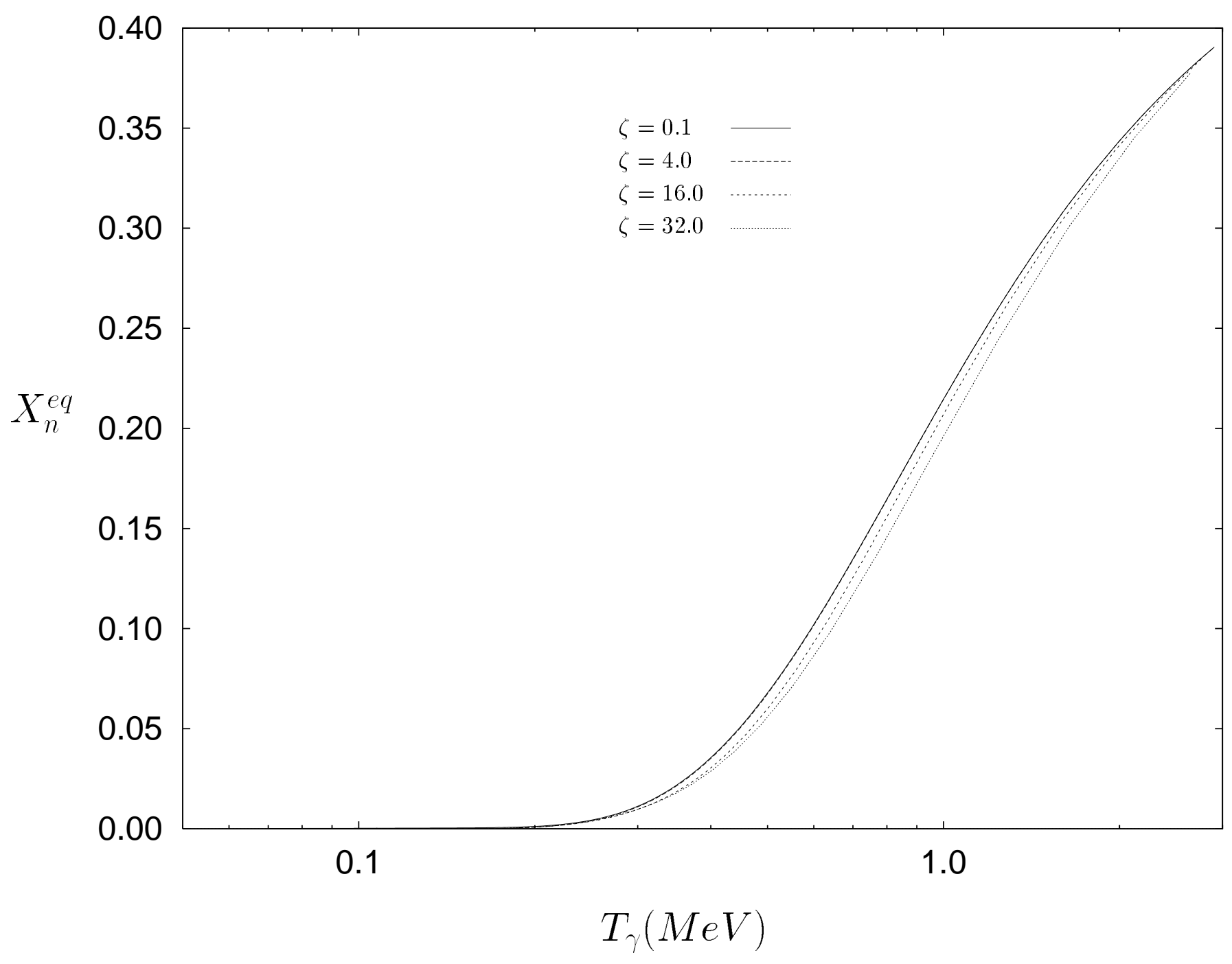

Figure 1 


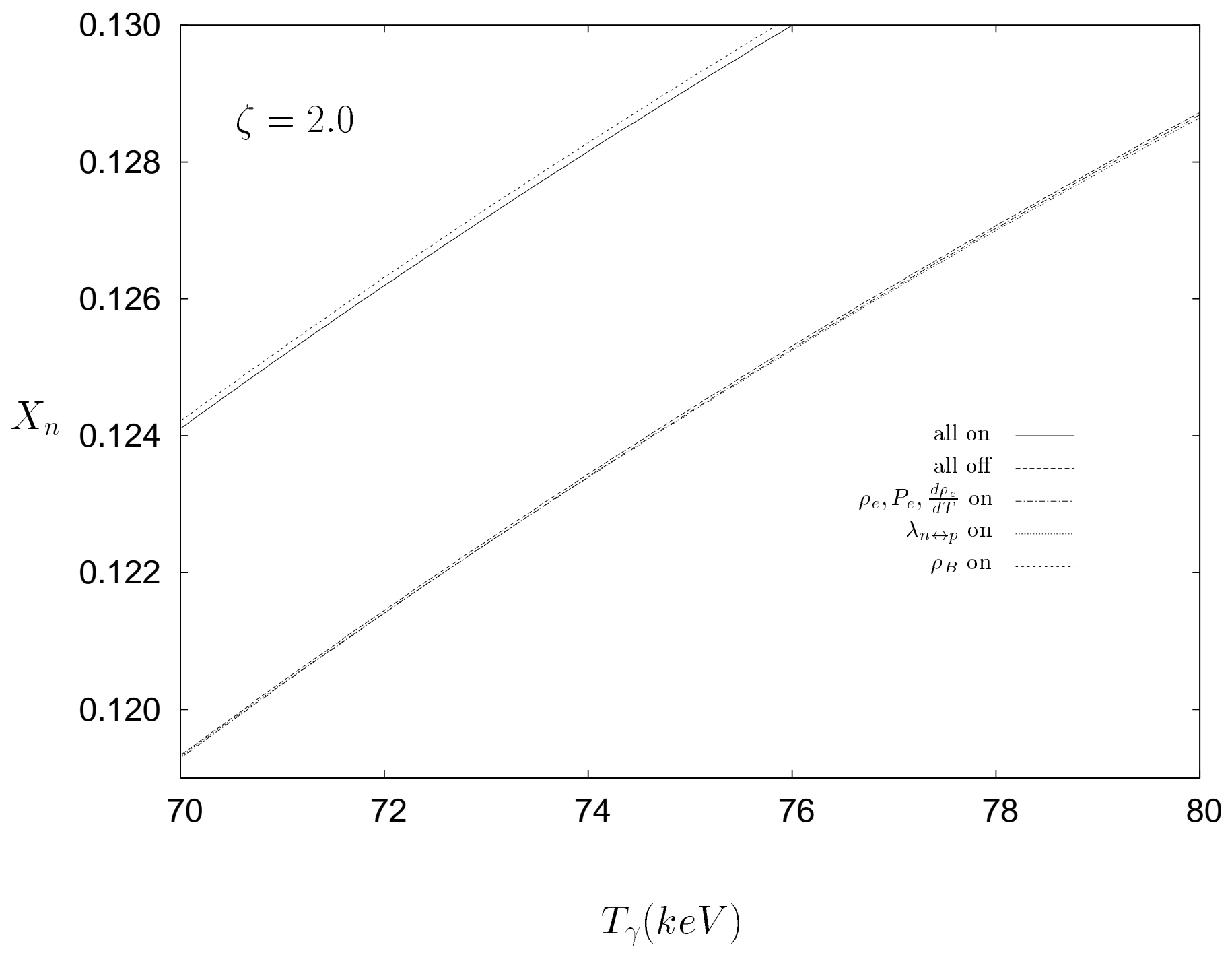

Figure 2 


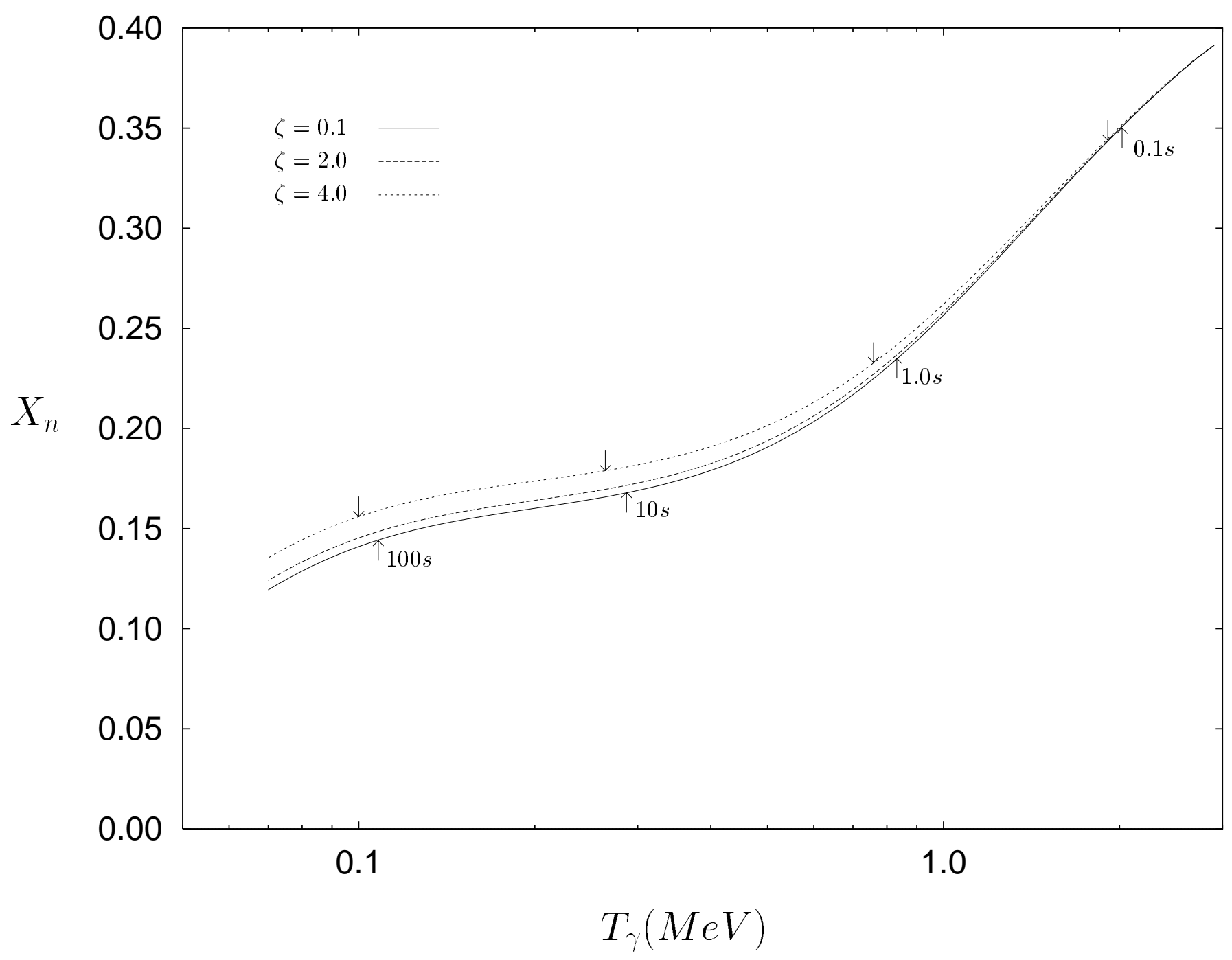

Figure 3 